\title{
Dynamic Modeling and Nonlinear Model Predictive Control of a Fluid Catalytic Cracking Unit
}

\author{
Raluca Roman ${ }^{\mathrm{a}}$, Zoltán K. Nagy, ${ }^{\mathrm{a}, \mathrm{b},}$, Frank Allgöwer ${ }^{\mathrm{b}}$ and Serban P. Agachi ${ }^{\mathrm{a}}$ \\ a'Department of Chemical Engineering, "Babes-Bolyai” University, 11 Arany Janos, \\ Cluj-Napoca, 3400, Romania \\ ${ }^{\mathrm{b}}$ Institute for Systems Theory in Engineering, University of Stuttgart, \\ Pfaffenwaldring 9, 70550, Stuttgart, Germany
}

\begin{abstract}
The paper presents the application of nonlinear model predictive control (NMPC) to a simulated industrial fluid catalytic cracking unit (FCCU). A complex dynamic model of the reactor-regenerator-fractionator system is developed and used in the controller. The novelty of the model consists in that besides the complex dynamics of the reactorregenerator system it also includes the dynamic model of the fractionator, as well as a new five lumped kinetic model for the riser, and hence it is able to predict the final production rate of the main products (gasoline and diesel). Simulation results presented in this paper demonstrate that a numerically efficient, inferential NMPC approach that regulates easily measurable variables in the reactor and regenerator is able to provide good control of the product composition for certain disturbance scenarios.
\end{abstract}

Keywords: catalytic cracking, nonlinear model predictive control, dynamic modeling.

\section{Introduction}

Fluid Catalytic Cracking Unit (FCCU) is one of the most important processes in a refinery, because its product is high quality gasoline. Due to its complexity, the modeling and control of FCCU poses important challenges (McFarlane et al., 1993). This chemical process has been traditionally controlled by using linear model predictive control approaches, which have proved their benefits in the petrochemical industries in the past two decades. Nonlinear model predictive control (NMPC) has the potential to achieve higher productivity by exploiting the advantages of taking process nonlinearities explicitly into account (Qin and Badgewell, 2003). However, the application of NMPC requires models with good prediction quality. In this paper simulation results obtained with a complex dynamic model of the FCCU are presented. The developed model simulates the dynamic behavior of the reactor-regeneratorfractionator system and predicts the composition of the main products (gasoline and diesel), which are then controlled in an inferential NMPC scheme, based on the complex high-order nonlinear model. The advantages of a modern NMPC approach, the so-called quasi-infinite-horizon nonlinear model predictive control (QIHNMPC) are

\footnotetext{
*Author to whom correspondence should be addressed: nagy@ist.uni-stuttgart.de
} 
illustrated to achieve better control performance, however with increased computational burden. Efficient solution of the on-line optimization is achieved even in the case of the very high dimensional model, based on a multiple shooting technique.

The paper is structured as follows: Section 2 presents the description of the plant and simulation results obtained with the model. Before conclusions, section 3 describes the NMPC approaches and optimization technique, with the simulation results.

\section{Dynamic Modeling of the FCCU}

The schematic diagram of the FCCU, for which the mathematical model was developed and the assessment of the NMPC has been performed is presented on Figure 1. In the FCCU raw material is mixed with the regenerated catalyst in the reactor riser. The cracking reactions and coke formation occur in the riser and the products (gasoline, diesel, slurry) are separated in a fractionator. The deactivated catalyst due to coke deposition is regenerated in the regenerator. The developed dynamic simulator consists of detailed models of: the feed and preheat system, reactor stripper, riser, regenerator, air blower, wet gas compressor, catalyst circulation lines and main fractionator. Based on the assumption given in Dupain at al. (2003) a five lump kinetic model (schematically shown on Figure 2) that predicts the yields of valuable products is proposed and included in the simulator. The resulted global model of the FCCU is described by a complex system of partial-differential-equations, which was solved by discretizing the kinetic models in the riser and regenerator on a fixed grid along the height of the units, using finite differences. The resulted model is a very high order DAE, with 2143 ODEs (143 from material and energy balances and 2000 resulted from the discretization of the kinetic models). The model was implemented in $\mathrm{C}$ programming language for efficient solution and was used first to study the dynamics of the process and then in the NMPC controller.

Figure 3 shows that the catalyst-oil ratio (CTO) has a small influence on the gasoline composition at the outlet from the riser, however it has an important influence on the composition in diesel of the product resulted in the riser. Therefore controlling the CTO is important in the plant. The model was also used to study the open-loop dynamic behavior of the system in the case of different disturbance scenarios. Figure 4, for example, illustrates the simulation results in the case of disturbance in the pressure drop between main the fractionator and the reactor.

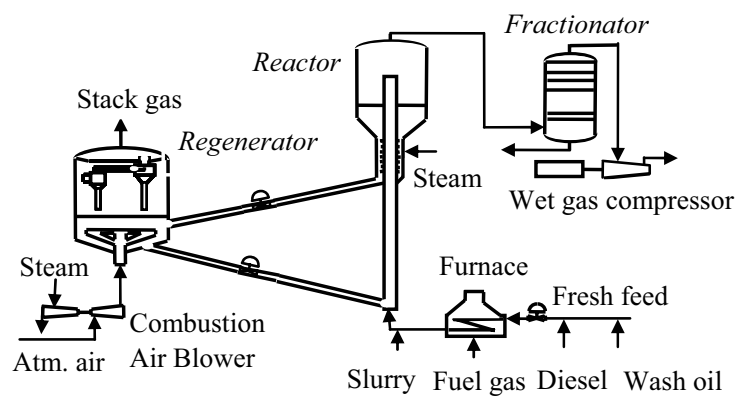

Figure 1. FCCU plant

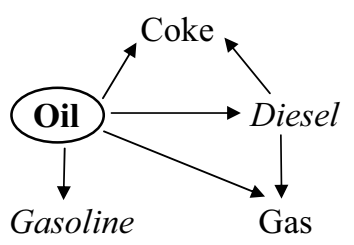

Figure 2. Five lump model for the catalytic cracking 

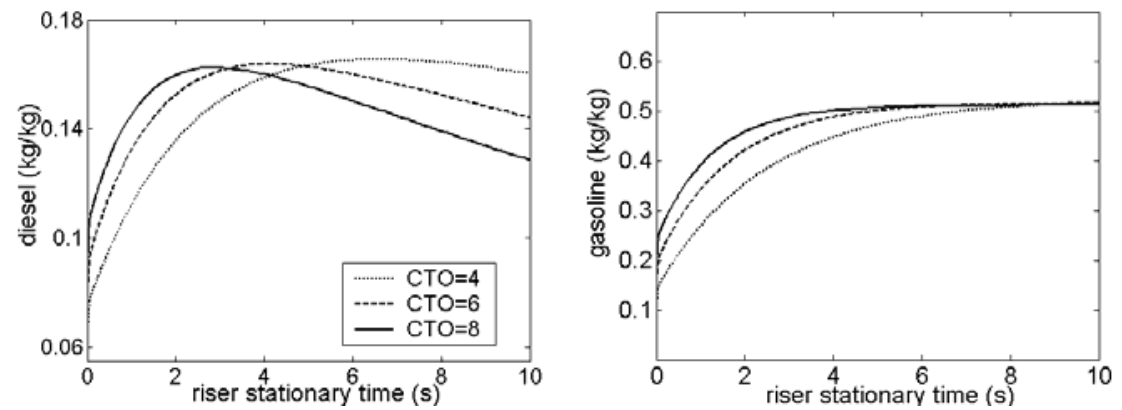

Figure 3. Composition profiles of the main products in riser at different catalyst-oil ratio (CTO)
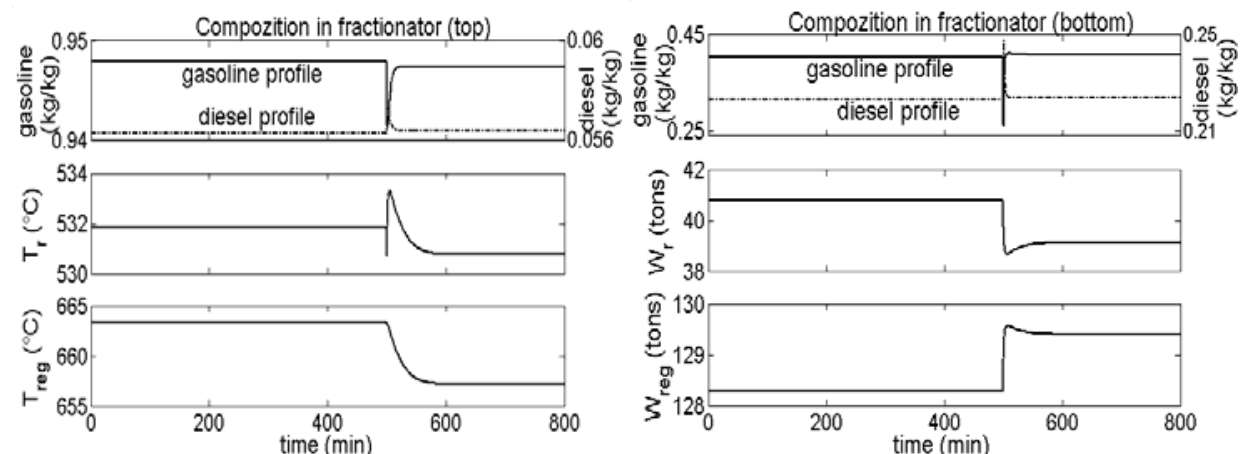

Figure 4. FCCU simulation results in the presence of pressure drop disturbance (10\% increase)

When the pressure drop increases with $10 \%$ at $t=500 \mathrm{~min}$, the temperature in the reactor $\left(T_{r}\right)$ and in the regenerator $\left(T_{r e g}\right)$ decrease $\left(1^{\circ} \mathrm{C}\right.$ for $T_{r}$ and $8{ }^{\circ} \mathrm{C}$ for $\left.T_{r e g}\right)$. This disturbance has a significant influence on the catalyst inventory: $6 \%$ increase in the amount in the reactor $\left(W_{r}\right)$ and $2 \%$ decrease in the regenerator $\left(W_{\text {reg }}\right)$, respectively. The influence of this disturbance for the gasoline and diesel composition in the fractionator is below $1 \%$, however considering the throughput of a typical FCCU this can lead to important economical consequences. The system is very stiff (there are large differences in the time constants of different process variables). In addition significant delays and complex dynamic behavior can be observed (e.g., higher order with zeros for the $T_{r}$ and non-minimum phase behavior for the gasoline composition in the bottom of the column), suggesting the use of NMPC, which is a control strategy that simultaneously can cope with complex nonlinearities, delays, and constraints, whereas optimizing economic control objectives.

\section{Nonlinear Model Predictive Control of the FCCU}

Nonlinear model predictive control is an optimization-based multivariable constrained control technique that uses a nonlinear dynamic process model for the prediction of the process outputs. Different NMPC approaches have been proposed that guarantee stability of the closed-loop system even under finite prediction horizon (Allgoewer et al., 1999). The approach used in this paper is the so-called quasi-infinite horizon 
nonlinear MPC (QIHNMPC) proposed by Chen and Allgoewer (1998). The basic idea of this approach consists of the approximation of the infinite horizon prediction to achieve closed-loop stability, whereas the input function to be determined on-line is of finite horizon only. The terminal penalty term is determined off-line. Using a local linear feedback low and a quadratic objective function, the terminal penalty term can be chosen to be quadratic. The basic formulation of the on-line control problem in the QIHNMPC can be expressed as below:

$\min _{u(\cdot)}\left\{\int_{t}^{t+T_{p}}\left(\|x(\tau ; x(t), t)\|_{Q}^{2}+\|u(\tau)\|_{R}^{2}\right) d \tau+\|\left(x\left(t+T_{p} ; x(t), t \|_{P}^{2}\right\}\right.\right.$

subject to: $\quad \frac{d x}{d t}=f(x, u), \quad x(t ; x(t), t)=\hat{x}(t)$

$$
\begin{aligned}
& x(\tau, x(t)) \in \mathcal{X}, u(\tau) \in \mathcal{U} \\
& x\left(t+T_{p} ; x(t), t\right) \in \Omega
\end{aligned}
$$

where $u(\tau) \in \mathcal{U}$ is the input vector, $x(\tau, x(t)) \in \mathcal{X}$ is the state vector, $\tau \in\left(t, t+T_{P}\right)$ is the time, $Q \in \mathbb{R}^{n \times n}$ and $R \in \mathbb{R}^{m \times m}$ denote weighting matrices, $T_{p}$ is the finite prediction horizon, $x(\cdot, x(t), t)$ is the trajectory given by the integration of the equation (4) driven by $u(\cdot):\left[t, T_{p}\right] \in \mathcal{U},\|x\|_{Q}^{2}=x^{T} Q x$ is the weighted 2-norm of $x$, and $\hat{x}(t)$ is the measured or estimated initial states. The terminal inequality constraint (4) with the last penalty term from (1) will force the states at the end of the finite prediction horizon to be in some neighborhood $\Omega$ of the origin called terminal region, which can be obtained by the iterative solution of a complex nonlinear optimization problem. In the case of the very complex FCCU, the determination of $\Omega$ is a nontrivial task, but since computation is performed off-line, it does not affect the real-time feasibility of the approach.

A very efficient solution technique for the problem (1)-(4) is based on the multiple shooting approach (Diehl, 2001). This procedure consists of dividing up the time interval $\tau \in\left[t, t+T_{p}\right]$ into a series of grid points $\left[t_{0}, t_{1}, t_{2}, \ldots, t_{f}\right]$ with $t_{0}=t$ and $t_{f}=t+T_{p}$. Using a local control parameterizations a shooting method is performed between successive grid points. The differential equations and cost on these intervals are integrated independently during each optimization iteration, based on the current guess of the control. The continuity/consistency of the final state trajectory at the end of the optimization is enforced by adding consistency constraints to the resulted nonlinear programming problem (NLP). A set of starting values for the state and adjoint vectors is required at each grid point in time, and continuity conditions for the solution trajectory introduce additional interior boundary conditions, which are incorporated into one large zero-finding problem to be solved. The solution of control problem is performed using an NMPC tool (Nagy et al., 2004) based on the sequential-quadraticprogramming (SQP) type optimizer HQP, which is used in conjunction with the implicit differential-algebraic-equation (DAE) solver, DASPK, for robust and fast solution of the model equations. Using this implementation, the computational time for the solution of one open-loop optimization is obtained below 2 min (the considered sampling time) even in the case of $2124^{\text {th }}$ order FCCU model.

The complex first-principles dynamic model was used in the NMPC algorithm. First the nominal NMPC is considered without the penalty term and terminal constraints, to test 

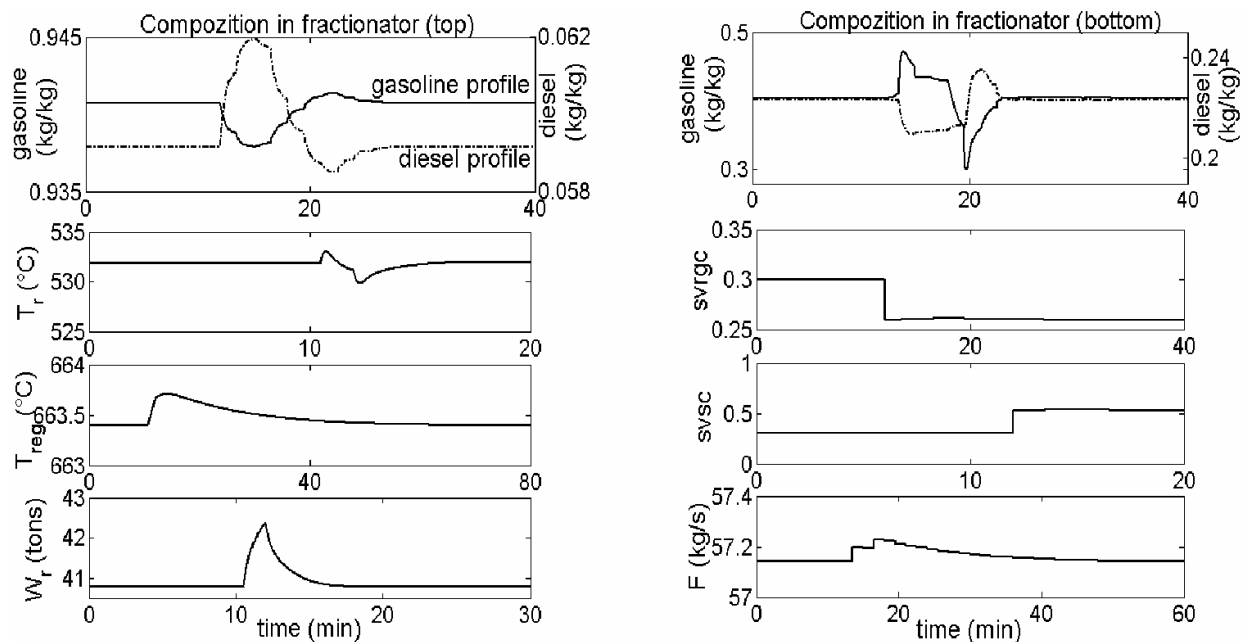

Figure 5. NMPC simulation results in the presence of pressure drop disturbance (10\% decrease at $t=12 \mathrm{~min})$. Different time scales are used to show the detail.
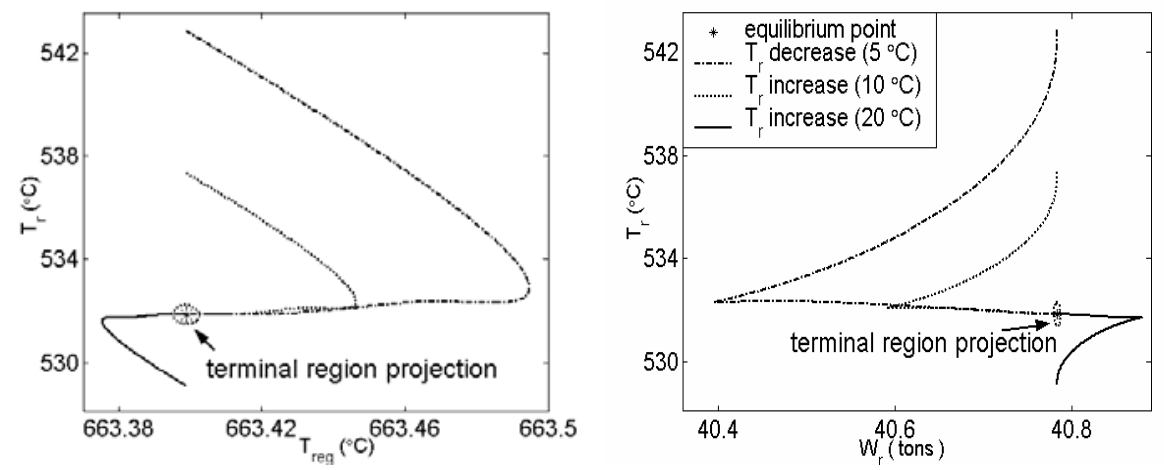

Figure 6. Trajectories for the system controlled by QIHNMPC

different control structures under different disturbance scenarios. From the simulations performed the best control structure resulted with the three controlled variables: reactor temperature $\left(T_{r}\right)$, regenerator temperature (Treg) and catalyst amount in the reactor $\left(W_{r}\right)$, and three manipulated inputs: openings of the spent and regenerated catalyst circulation pipes between the reactor and regenerator ( $s v s c$ and $s v r g c$, respectively) and the flow rate of the raw material $(F)$. This inferential control scheme is able to provide good control performance for the composition in the fractionator (see Figure 5). Figure 6 illustrates the performance of the QIHNMPC for different off-nominal initial conditions. It can be shown that asymptotic stability is achieved in all cases. The very small terminal region (projections of the hyper-ellipsoid on the shown state space) is caused by the strong nonlinearity of the system. Figure 7 illustrates that QIHNMPC achieve better control performance than NMPC. Using QIHNMP the system is stabilized faster. 

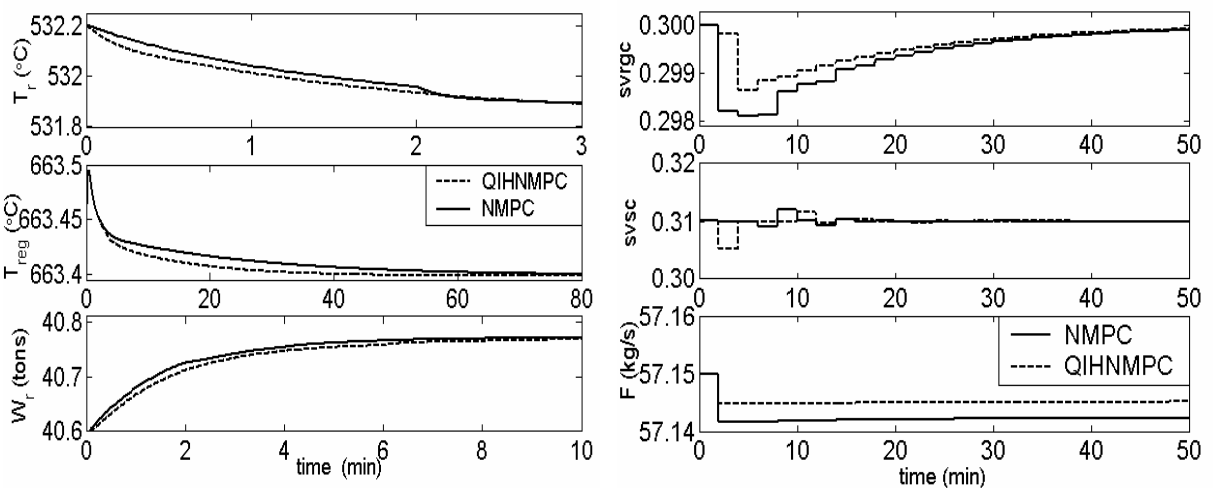

Figure 7. Comparison between QIHNMPC and NMPC; variation in time for $20^{\circ} \mathrm{C}$ disturbance in $T_{r}$ (dashed line in Figure 6); Left - controlled outputs; right - manipulated inputs

\section{Conclusions}

The paper presents dynamic simulations for the FCCU aggregate system that includes the main fractionator and a kinetic model for the riser leading to a $2144^{\text {th }}$ order ODE model. Based on this model an inferential control scheme is proposed that is able to control the product distribution resulted from the fractionator based on easily measurable variables in the regenerator-reactor system. The model was used to simulate the performance of the theoretically founded quasi-infinite-horizon NMPC (QINMPC), to achieve fast stabilization of the closed-loop system. It is shown that using state-ofthe-art optimization approaches based on modern multiple shooting algorithm real-time feasibility can be achieved even in the case of the very high order FCCU model. The results demonstrate that industrial applications of modern NMPC approaches to complex chemical processes can be brought in the realm of possibility.

\section{Acknowledgement}

This work was supported by the Marie Curie fellowship HPMT-CT-2001-00278.

\section{References}

Allgoewer F., T.A. Badgwell, J.S. Quin, J.B. Rawlings, and S.J. Wright, 1999, Nonlinear predictive control and moving horizon estimation-An introductory overview, In P.M. Frank (editor), Advances in Control, 391.

Chen H and F. Allgoewer, 1998, A Quasy-Infinite Horizon Nonlinear model Predictive Control Scheme with Guaranteed Stability, Automatica, 34, 1205.

Diehl M., Real-Time Optimization for Large Scale Nonlinear Processes, 2001, PhD Thesis, University of Heidelberg.

Dupain X, E. D. Gamas, R. Madon, C.P. Kelkar, M. Makkee, J.A. Moulijin, 2003, Aromatic gas oil cracking under realistic FCC conditions in a microriser reactor, Fuel, 82, 1559.

Qin, S.J., and T. Badgewell, 2003, A Survey of Industrial Model Predictive Control Technology, Control Engineering Practice, 11, 733.

McFarlane R.C., R.C. Rieneman, J.F. Bartee and C. Georgakis, 1993, Dynamic simulator for a model IV Fluid Catalytic Cracking Unit, Computers Chem. Engng, 17, 275.

Nagy Z. K., F. Allgower, R. Franke, A. Frick, B. Mahn, 2004, Efficient tool for nonlinear model predictive control of batch processes, in Proc. of the 12th Mediterranean Conference on Control and Automation (MED'04), Kusadasi, Turkey, on CD. 